\title{
Valoración e implementación de la estrategia pedagógica para la caligrafía en la formación del maestro primario
}

\author{
Dr. María Lescaille Rivera \\ mlescailler@udg.edu.cu \\ Orcid. 0000-0001-7420-2340 \\ Universidad de Guantánamo. Cuba \\ Dr. Milton Doroteo Cayambe Guachilema \\ Cayambe1970@hotmail.com \\ Orcid.org/0000-0003-4772-894X \\ Universidad de Guayaquil \\ Dr. Felipe Manuel León Cáceres \\ manuel_leonc@hotmail.com \\ Orcid. 0000-0003-4760-9342 \\ Instituto Superior Universitario \\ Bolivariano de Tecnología \\ MSc. Douglas Mario Alvarado Herrera \\ domaalhe@gmail.com \\ Orcid. 0000-0001-9203-7975 \\ Universidad de Guayaquil \\ MSc. Manuel Pedro Palacios Noboa \\ peter_pala@msn.com \\ Orcid. 0000-0003-0244-4708 \\ Universidad de Guayaquil \\ MSc. Ronny Cesar Alvarado Pincay \\ ronnyalbapin@gmail.com \\ Orcid. 0000-0002-3958-9778 \\ Universidad de Guayaquil
}

\section{RESUMEN}

La formación inicial de los maestros primarios en Cuba, tiene como propósito lograr un egresado capaz de alcanzar una preparación de excelencia para su futuro desempeño profesional. Sin embargo, las problemáticas de los estudiantes referida a la caligrafía, revelan en ocasiones, situaciones críticas en su escritura que posibilitan determinar el objetivo como: la valoración e instrumentación de una estrategia pedagógica para la caligrafía en la formación del maestro primario. La metodología seleccionada constató las dificultades a partir de la observación a clases, revisión de cuadernos, prueba escrita 
a los estudiantes y entrevista a los docentes, estas últimas demostraron que los discentes tienen limitaciones en el uso de los trazos de grafemas, enlaces entre otros. La implementación de la estrategia demostró que el tratamiento sistemático ofrecido por varios docentes, corroboró el salto cualitativo y cuantitativo de los estudiantes de la carrera, constatado en las visitas al componente laboral de los futuros maestros, si no en los exámenes escritos evaluados durante 4 cursos académicos y la entrega de trabajos independientes. Además, otros resultados permitieron que un total de 8 estudiantes de la muestra seleccionada, participaran en Concursos a nivel de universidad, y Festivales de clases, demostrando mejores rasgos caligráficos.

Palabras clave. caligrafía; formación; estudiantes; estrategia; evaluación. 


\title{
Valuation and implementation of a calligraphic pedagogical strategy in the training of elementary teachers
}

\begin{abstract}
The initial training of elementary teachers in Cuba has as its main propose, the preparation of a competent graduate, who is able to carry out his/ her future professional work with Excellency. Nevertheless, these teachers-to- be reveal problems with their calligraphy, which in occasions have to do with difficulties with their writing. All these led to the determination of the following objective: to valuate and put into practice a calligraphic didactic strategy in the training of elementary teachers. The methodology chosen helped to verify these difficulties, out of class observations, revision of the future teachers' notebooks, written exams, and interviews to their teacher- trainers. These latters demonstrated that teachers-to-be have limitations with their handwriting, the tracing of graphemes, or lettering. The implementation of the strategy proved that the systematic treatment done by several teacher-trainers, corroborated the qualitative and quantitative changes undergone by the students of this major, not only in their teachers practicum class observations, but in their written exams during four academic years, as well as in their independent works. Besides, other outcomes allowed eight future teachers of the sample take part in university contests, class festivals, where they showed better calligraphic skills.
\end{abstract}

Key words: calligraphy; training; students; strategy; valuation.

Artículo recibido: 15 noviembre. 2021 Aceptado para publicación: 10 diciembre 2021 Correspondencia: mlescailler@udg.edu.cu Conflictos de Interés: Ninguna que declarar 


\section{INTRODUCCIÓN}

Los cambios sociales repercuten en los sistemas e instituciones educacionales, porque reclaman de un nuevo sujeto social y profesional, capaz de desempeñarse en correspondencia con las características del paradigma cultural inaugurado en este milenio: la era del conocimiento, cuyas tres características básicas son: los cambios vertiginosos y sucesivos, la revolución científico-tecnológica, y la universalización de los fenómenos socioeconómicos y político-culturales. Por tanto, se requiere de niveles superiores corroborando la capacidad intelectual, creatividad e innovación, y una aptitud personal para aplicarla.

Una forma de conjugar la participación multidisciplinar en la solución de problemas se encuentra en los planeamientos estratégicos, lo que ha generado un nuevo enfoque: El enfoque estratégico que según, González, C. (2007)

... “implica nuevas concepciones educativas, organizativas y de gestión. La proyección es imprescindible y la solución de problemas tiene un sentido nuevo, que encamina a las personas hacia cambios sustanciales para que se liberen de suposiciones tradicionales. La visualización invita a percibir la organización bajo una nueva óptica y las instituciones educacionales tienen estas características: son flexibles, en contacto con el medio laboral y social, satisfacen las demandas formativas de una sociedad con acelerado ritmo de innovación científico-tecnológica y universalización de los fenómenos, cuyo capital intelectual es clave de la productividad y competitividad, ofrecen formación educativa pertinente, con calidad y equidad social, y forman educandos competentes, civil y profesionalmente, con perfil laboral básico, polivalente y multifuncional, y dispuestos al aprendizaje permanente."

Según criterios de esta autora, la única actitud personal y profesional válida es la que opta por el aprendizaje innovador, en la que la anticipación y la participación son gestiones para concebir y conducir, mediante proyectos innovadores, las acciones educativas transformadoras, con pensamiento sistémico, planeación estratégica abierta, flexible, participativa, contextualizada, imaginativa y visionaria. 
En este sentido, es justo precisar que el sistema educativo cubano es centralizado y dentro de él, el modelo caligráfico debe ser apropiado por los ciudadanos, porque facilita el desarrollo de las funciones de la escuela, de los profesores y maestros primarios, que deben concebir planes, programas, estrategias, orientaciones, que posibiliten la flexibilidad asumida como principio, lo que ha permitido propiciar cierta descentralización en la práctica educativa, con mayor fuerza en el nivel universitario.

En la escuela y mucho más en niveles educacionales superiores, se ha constatado como una problemática a resolver la caligrafía, la cual ha quedado reducida a patrimonio de la disciplina Lengua Española y Lenguaje y Comunicación así como la Metodología de la Enseñanza de la Lengua Materna (MELE) en la formación de maestros primarios. El Programa Director de Lengua Materna (PDLM) permite una descentralización del tratamiento al componente caligráfico como habilidad comunicativa desde la interdisciplinariedad, como tendencia en las relaciones científico- metodológicas, pero no se sistematiza la atención por todos los docentes ni en todos los contextos, de ahí la brecha investigativa.

Por eso, como propósito resulta necesario retomar fundamentos teóricos acerca de la historia de la caligrafía de la Lengua Española, marcada por una evolución estética desde el jeroglífico hasta la escritura alfabética arábiga que ha llegado hasta nuestros días. En la actualidad se restringe su enseñanza a dos estilos básicos: script y cursivo, de los cuales hay infinidad de modelos en las Tecnologías de la Información y las Comunicaciones (TIC) y diversos criterios sobre la conveniencia de uno u otro en el desempeño humano. El mayor auge se ubica en la Edad Media donde proliferaron publicaciones en forma de álbumes, repertorios y manuales. Fue regida durante mucho tiempo por la iglesia y la primera investigación sobre la misma, se realizó por el británico Edward, J. (1906). Por eso, a raíz del siglo XX creció el interés por su utilidad y su enseñanza- aprendizaje.

Desde la ciencia ha tenido diversas miradas, según las posiciones del investigador: Edward, J. (1906) como decoración e iluminación en el diseño y la arquitectura; Freeman (1945), estudia el movimiento de los escolares de la escritura script a la cursiva; Berstein (1947) y Luria, A. (1982) realizan importantes estudios sobre su base neuromotriz; Vygostky, L. (1966), su adquisición en la edad infantil y su papel en la transmisión del pensamiento; aspectos de gran significación para la escritura. 
La investigadora nacional Roméu, A. (2003) sistematiza investigaciones de autores como: Diringer (1968),quien la define y precisa como habilidad: Navarini (1972) alude a sus niveles de uso determinándolos como funciones lingüísticas y coincide con el criterio anterior, al destacar su utilidad tanto como la palabra hablada; Brucckerner y Bond ( 1975); destacan el uso de cada estilo en sus estudios sobre diagnóstico, así como el tratamiento a las dificultades en el aprendizaje; en los estudios acerca de la lengua y su didáctica, Halliday (1973) y Coulmas (1989), distinguen entre el uso heurístico y el imaginativo, lo que da cuenta de la variedad de criterios al respecto y fundamentan la necesidad de su adecuado tratamiento en la formación inicial del maestro primario.

En Cuba, destacados estudiosos abordaron las primeras ordenanzas en el siglo XIX Las Casas, Luz y Caballero, escribió las primeras indicaciones metodológicas; Alfredo Morales y Morales (1879) elabora una Cartilla de alfabetización. Posteriormente, Alfredo Miguel Aguayo (1923), destaca su utilidad para la formación estética. Por su parte, la elaboración de orientaciones y propuestas metodológicas estuvieron centradas en Almendros, H. (1961) González, R. (1983); Lescaille, M. (-2015) y Domínguez, I. (2008) entre otros quienes ofrecieron sus preceptos normativos en estas últimas décadas.

Con respecto a las definiciones de caligrafía son diversas (del griego kaligraphia, escritura bonita). Según diccionarios y enciclopedias, es "el arte de escribir con letra hermosa, pulcra, bella y correctamente formada, según las normas y estilos así como con el conjunto de rasgos que caracterizan la escritura de una persona, de un documento". Estudios contemporáneos amplían la definición destacando su papel en la comunicación y el arte: (Mediavilla, 1996), Cardeso, Mulet, Y. (2008), Espinosa Janeiro (2009). Se asume la ofrecida por Luria (1982) cuando alude: "La caligrafía se caracteriza como un movimiento voluntario (praxis), en su realización se dan coordinaciones entre sistemas, que se corresponden con la definición de este tipo de movimiento y constituye un sistema funcional complejo, que se sustentan en lo biológico, neurológico y anatomofisiológicos influyendo en las coordinaciones óculo y psico -motrices necesarias para la realización correcta".

Lo anterior permite incluir actividad caligráfica, dentro de la manipulación compleja de objetos ya que el manejo del lápiz o la pluma, requieren una compleja coordinación de impulsos descendentes y ascendentes, giratorios, oscilantes de resistencia y continuidad, que no ocurre con otras manipulaciones de objetos, por lo tanto, no está exenta de ser 
alterada por lesiones, o patologías, no solo de diferentes áreas corticales, sino también en los órganos de las manos: articulaciones, huesos, ligamentos, etc. ya sea por causas degenerativas, como por traumatismos de origen exterior o agresiones ambientales, como de base neurológica o neurofuncional.

Desde esta perspectiva, diferentes ciencias han desarrollado estudios acerca de la escritura y la caligrafía, lo que permite ofrecer una mirada holística a su significación. Su estudio ha propiciado el surgimiento de diversas ciencias como la grafología, criptografía, epigrafía, entre otras. También son variados los aportes que valoran sus potencialidades como base investigativa: la lingüística, el psicoanálisis, la neurología, la antropología, la criminalística y otras, pues es una huella singular que deja la persona sobre la cual se pueden realizar muchas inferencias gnósicas. Por eso, el propósito del artículo es ofrecer un aporte práctico que permita solucionar las problemáticas de los estudiantes de carreras pedagógicas en la educación superior.

En la región la investigadora Susana B González (2005) pedagoga colombiana refiere que realizó un estudio del estado de la caligrafía en los estudiantes del nivel medio en su país y encontró que "alrededor del $75 \%$ de sus alumnos escriben con letra de imprenta, aún más lamentable descubrir que muchos de ellos no saben hacerlo de otra forma" de modo que destaca la relación escritura, lectura y comprensión jerarquizando el papel de la primaria en el logro de las otras habilidades. Ella plantea que: “el niño es un gran imitador que además sabe distinguir por intuición las formas bellas o armoniosas de las que no lo son". Por esta razón el docente debe comenzar a encauzar, en él, un juicio crítico y estético. Valora como necesario que el maestro asuma una postura ejemplar, normativa y enriquecedora.

Otros autores regionales de Ecuador Cayambe, Palacios, Alvarado y león (2021) refieren que arriban a la Educación Superior estudiantes con serias dificultades en su escritura, y no se percatan que esto afecta su calidad como futuros profesionales, aunque se gradúen de tecnólogos. Asimismo, en Uruguay Mir (2006) y Argentina, Castañeda (2010) corroboran con aisladas investigaciones, que los estudiantes llegan con problemas en el uso de la lengua oral y escrita a su juventud, lo que entorpece la calidad de su competencia comunicativa tan vital en un egresado.

Por consiguiente, la estrategia pedagógica que se propone está dirigida a la coordinación de acciones concebidas para la funcionalidad del sustento teórico, donde su categoría 
fundamental es la actuación caligráfica profesional contextualizada, e incluye la implementación del procedimiento rehabilitación caligráfica prospectiva, desde una perspectiva clínico- pedagógica y con enfoque cognitivo, comunicativo y sociocultural. La revisión métodos y variedad de textos bibliográficos posibilita adentrarse en el siguiente epígrafe.

\section{MATERIALES Y MÉTODOS}

Se realizó un estudio pre- experimental de tipo pedagógico utilizando el método de investigación acción, por su carácter participativo. Es una investigación cualitativa en tanto el cambio que se busca en la actitud de los sujetos debe tributar al cambio en la actuación y por tanto certifican la hipótesis. Algunas características de la investigación acción participativa fueron tenidas en cuenta: es una modalidad investigativa en el que el objeto se convierte en sujeto; lo cual posibilita el protagonismo grupal; además, es democrático porque colaboran investigadores y participantes, da mayor peso a lo cualitativo, a la comunicación interpersonal y con ello las relaciones interpersonales son de diálogo y entendimiento, se pone al servicio de grupos y categorías sociales desfavorecidas y la evaluación es cooperativa entre todos los participantes.

Objetivo del pre- experimento: promover el desarrollo profesional del maestro primario en formación para que puedan desempeñarse con integralidad en la escuela primaria.

Pero para ello, se requiere ofrecer superación profesional a docentes que laboran en la formación de estudiantes de la carrera Educación. Primaria con un curso de postgrado, que le brinde las herramientas teórico- pedagógicas necesarias para su mejor abordaje en el proceso educativo. Su esencia está en organizar el tratamiento a la caligrafía con un enfoque más actualizado a partir de asumir las nuevas tendencias que figuran en la enseñanza cubana actual, referida al enfoque comunicativo, cognitivo y sociocultural que brinda una mayor atención a los procesos psicológicos y el desarrollo intelectual convirtiéndose en un proceso más desarrollador.

Es decir, un enfoque histórico cultural donde se retome la historia de la caligrafía, su valor caracterizador y de diagnóstico, sus valores formativos e instructivos y otros, enmarcado en el momento histórico y en el saber acumulado por el maestro primario en formación. . Asimismo refleja un enfoque comunicativo profesional pedagógico desde la caligrafía, basado en el desarrollo de habilidades comunicativas orales y escritas.

El pre-experimento se estructura en tres etapas que se describen a continuación: 
Etapa 1: Diagnóstico de partida que persigue elaborar un estado inicial de las variables que se someterán a proceso y poder constatar su comportamiento antes del preexperimento. Se aplicó un cuestionario y una prueba pedagógica.

Etapa 2: Proceso de pre- experimentación, consistió en aplicar el modelo diseñado en el tiempo asignado en el programa y módulo para el tratamiento al componente caligráfico.

Etapa 3: Diagnóstico de cierre con el fin de poder valorar a partir de un proceso comparativo la validez de los resultados obtenidos. Para ello se reaplicaron los instrumentos entrevista, pruebas pedagógicas para la elaboración del diagnóstico de entrada.

Se constató la eficiencia que se fue logrando en cada etapa declarada para el tratamiento a la caligrafía. Así como la solución revelada a partir de la solución a las dificultades en el tratamiento a la caligrafía del maestro primario en formación, que limitan la adquisición de habilidades para su desempeño profesional.

Como variables se determinan: Actuación caligráfica profesional, donde los indicadores son: Dominio de elementos teóricos. Dominio de las cualidades caligráficas y Habilidades comunicativas.

Definición operacional: Se someten a pre- experimentación los siguientes elementos. La secuencia lógica del tratamiento, las actividades diseñadas, las orientaciones para cada etapa y el sistema de indicadores para la evaluación y diagnóstico.

La población la conforman 56 maestros primarios en formación pertenecientes a los cursos del 2016 - 2020 seleccionados por no ser las cohortes ingresadas en ese período. Siendo la muestra coincidente con el $100 \%$ de la población. El grupo de control 25 maestros primarios en formación seleccionados aleatoriamente, se escogen a partir de tener las mismas características en el nivel de partida y ser suficientes estadísticamente en cuanto a número de individuos que el grupo experimental, pues constituyen el $48 \%$ de la población

La población de profesores estuvo integrada por 32 profesores del departamento carrera de Licenciatura en Educación. Primaria y se selecciona como muestra 15 profesores de la disciplina Estudios Literarios y Metodología de la enseñanza de la lengua materna en la escuela primaria (MELE) que representan el 46, 8\% de la población, mucho más representativa. 
De ellos solamente cinco son graduados de la especialidad maestros primarios; una, es filóloga y el resto, son graduados de Español - literatura, es decir especialistas de estas asignaturas. Todos pasan de 10 años de experiencia en la docencia en la Universidad y más de 15 en la carrera Licenciatura en Educación Primaria; cuatro de ellos se sometieron a rotación por la enseñanza primaria como antesala de las transformaciones educacionales que se avecinaban. De ellos, dos fueron jefas de departamento y tres jefes de la disciplina. Un elemento común entre todos y distintivo al mismo tiempo, es que ninguno recibió caligrafía durante su formación como profesionales. El 70 \% ciento no usa la escritura modelo de la escuela primaria en sus clases. Otras técnicas de los métodos empíricos fueron: pruebas pedagógicas, medios de enseñanza de la caligrafía, entrevista, criterios de especialistas y de usuarios junto al pilotaje.

\section{Resultados y discusión del pre-experimento.}

Etapa 1: En la encuesta aplicada en el diagnóstico de entrada se observó que algunos estudiantes conocían que las letras son un todo y que cada una de ellas está formada por pequeñas partes denominadas rasgos, de lo que se infiere que no dominaban sobre rasgos, trazado, enlaces y formas. Todos los estudiantes sabían de forma elemental y aproximada el concepto de caligrafía y la reconocen como una habilidad profesional para el maestro. En la realización del pre- experimento se procedió a crear las condiciones para la aplicación de los modelos de ejercicios docentes, lo que se planeó de forma estratégica, teniendo en cuenta las dimensiones más simples a las más complejas, es decir, de lo externo a lo interno. Se aplicó la estrategia a un total de 25 estudiantes de primer año de la carrera Licenciatura en Educación Primaria en correspondencia directa con el criterio de selección definido: Ser estudiantes del primer año de la carrera. Estar bajo la influencia directa de la investigadora.

Para elaborar el diagnóstico inicial se realizó el análisis de una prueba pedagógica de entrada, consistente en un dictado. La variable fundamental fue el tratamiento a la caligrafía, a la que se desglosó en las cuatro cualidades básicas que se trabajan en la escuela primaria: uniformidad, fluidez, corrección, inclinación. Las tres primeras cualidades se evaluaron a partir de la apreciación de bien, regular o mal y se clasificaron como sigue:
$1=$ Mal
$2=$ Regular
y $3=$ Bien. 
La inclinación se evaluó de bien y mal solamente por ser una cualidad que se tiene o no, ya sea hacia la derecha o hacia la izquierda. La norma cubana de caligrafía establece la inclinación de la letra cursiva hacia la derecha. De ese modo 1 es Bien y 2 obtienen mal. La calidad de los escritos se midió según los indicadores establecidos por el Instituto Central de Ciencias Pedagógicas: legible, legible con problemas de trazos y enlaces, legible con problemas de trazos o enlaces e ilegible. Con los datos obtenidos se elaboró una tabla para registrar los datos de cada prueba de forma individual y se realizó un procesamiento estadístico con la aplicación del paquete computarizado. Se obtuvieron los siguientes resultados.

Las cuatro cualidades fueron medidas en los 45 estudiantes, lo que se refleja en la tabla de registro de frecuencia 1 . Se midió la presencia de cada cualidad por separado en los trabajos del estudiante. La uniformidad se comportó mal en 23 estudiantes para un 92,2 $\% ; 3$ de ellos, resultaron evaluados de regular para un $7.8 \%$ y ninguno de bien para un $0 \%$. Este resultado muestra una alta prevalencia de la falta de uniformidad en los escritos, lo que se considera una insuficiencia caligráfica que afecta la expresividad y estética en la escritura. La fluidez estuvo mal en un $92.2 \%$ y regular y mal en un $7,8 \%$, lo que revela la insuficiencia caligráfica.

La corrección estuvo mal en 23 estudiantes para un 92,2 \%. Este resultado, es de alta frecuencia y significativo por la magnitud de los errores cometidos en el trazado y enlace de las grafías en la letra cursiva, toda vez, que este tipo de error determina la comprensión lectora. La inclinación se comportó mal en un $7.8 \%$ de la muestra, lo que también es una alta frecuencia y significativo por la imagen de regresión que trasmite.

Finalmente se aplicó una evaluación general a cada estudiante, para lo cual se determinó considerar la presencia de entre 3 y 4 insuficiencias para evaluar de Mal; la presencia de 2 errores para regular y la presencia de hasta 1 error, para evaluar de bien, si y solo, si la cualidad afectada no fuera la inclinación, en caso tal, se evalúa de mal. De ello resultó de bien, un total de 2 estudiantes para un $8 \%$ que unidos a los 15 evaluados de regular los cuales reportan una frecuencia de incidencia de hasta el 93,3\%. Del análisis realizado se infiere que la cualidad más afectada fue la uniformidad, seguida de la fluidez y que la menos afectada era la inclinación. La evaluación justificó la necesidad de transformación de los modos de actuación mediante la estrategia pedagógica para la formación del maestro con énfasis en la caligrafía como componente de la lengua materna. . 
Durante el proceso de pre- experimentación se apreció un cambio sustantivo de actitud frente al aprendizaje da la caligrafía. Los estudiantes se mostraron interesados, deseosos de ir a la práctica laboral y se disputaban ir a la pizarra, fue significativo cómo los directivos de la Facultad y el Departamento apreciaron el cambio evidenciado pues se mostraban más estimulados por los saberes obtenidos.

Al concluir la aplicación que duró el primer módulo del primer año, se realizó otra medición de la frecuencia de presencia de las cualidades caligráficas en la escritura de los estudiantes. Se aplicó otra prueba pedagógica y se midió el comportamiento de las cualidades en cada estudiante individual y colectivamente. La uniformidad mejoró al resultar bien en 23 estudiantes para un 92,2 \%, y regular en el $0 \%$. Aquí se aprecia la no frecuencia en caso de evaluados de mal.

La fluidez estuvo bien en 23 estudiantes para un 92,2\%. Solo 2 estudiantes mantuvieron dificultad representativa del 2, $2 \%$. La corrección se comportó con igual resultado que las anteriores. La inclinación experimentó una mejoría sustancial al ser modificada toda la evidencia y resultar los 25 estudiantes en la categoría de bien, el $100 \%$. La evaluación general se realizó siguiendo los procedimientos anteriores y resultó que 23 estudiantes culminaron evaluados de bien para un $92,2 \%$, con una frecuencia acumulada entre bien y regular del $100 \%$. Las inferencias realizadas del análisis son:

La uniformidad se incrementó en 23 estudiantes evaluados de bien, lo que aumentó la frecuencia de su manifestación en los trabajos escritos. La fluidez se incrementó en 23 estudiantes evaluados de bien. La corrección mejoró en 23 estudiantes. La inclinación se manifestó en el 100 \% y la evaluación final se incrementó en 23 estudiantes con bien. Es decir que se reporta en ellos un salto cualitativamente superior en las insuficiencias caligráficas que dieron origen a la investigación. La suma de los estudiantes evaluados de bien y regular ofrece una apreciación de: Uniformidad 23 inicial; 2 final. La corrección idéntico resultado. Se pudo apreciar que el $100 \%$ de los estudiantes presentaban escritura legible.

La diferencia de 16 estudiantes con reporte de mejoras rotundas en su conducta caligráfica, permite considerar de muy adecuado el trabajo realizado y evaluar de válida la estrategia de rehabilitación diseñada. La encuesta aplicada para recoger la opinión de los estudiantes concluye con los siguientes resultados. Todos los Items fueron marcados por el $100 \%$ de los estudiantes. Se pudo constatar que todos los estudiantes despertaron 
expectativas, mejoraron su caligrafía, su actuación profesional, su gusto estético, dominaron el concepto de caligrafía, aprendieron a revisar libretas, y explicar el proceso de enseñanza de la escritura de una grafía y al logro de una cualidad.

Como aporte de la investigación, emergió el procedimiento Rehabilitación caligráfica prospectiva como una adecuación y aporte al método Análisis Discursivo Funcional del Enfoque Cognitivo, Comunicativo y Sociocultural de Roméu (2006). Este procedimiento se aplicó a través de un pre- experimento formativo en la formación inicial de maestros primarios de la Universidad de Guantánamo. El análisis realizado posibilita arribar a los siguientes resultados

\section{RESULTADOS Y DISCUSIÓN}

El postgrado ofrecido a los docentes seleccionados como parte de su superación ofreció los fundamentos teóricos y pedagógicos para brindar atención a las problemáticas de los estudiantes no solo en el proceso educativo y pedagógico, sino también en los distintos contextos educativos. Por ejemplo, el estudiante participa en el componente laboral donde no solo observa clases del maestro tutor, sino que también diseña clases e imparte en los dos últimos años clases a los escolares de la escuela primaria. De modo que se convierte en un modelo para sus discípulos en el uso de la lengua oral y escrita.

Asimismo, participa en el componente investigativo con ponencias para eventos, su tesis final de culminación de estudio y otras actividades culturales como concursos, presentación en festivales de clases, festivales culturales que revelan sus modos lingüísticos. De manera que es vital persuadir al estudiante de la necesidad imperiosa de solucionar los problemas de la lengua escrita.

Posterior a la culminación del postgrado con los docentes, se visitaron clases para constatar la implementación de estos referentes teóricos en las mismas, demostraron ser más dinámicas, emotivas y se elevó la comunicación en el grupo. La revisión de documentos de la carrera y de los docentes permitió constatar que los estudiantes aprendieron a usar el color, a realizar tirillas, participaron en concursos de caligrafía a nivel de Universidad.

Se elaboró un sitio Web en la carrera sobre la caligrafía y en general consideraron en un $100 \%$ que esta manera de aprender la caligrafía fue interesante, argumentaron que el aporte utilizado permitió apropiarse de contenidos, asumir sus dificultades, y la necesidad de desarrollar sus hábitos y habilidades como futuros maestros primarios, incluso 
advirtieron que la caligrafía y la escritura son habilidades a resolver durante la carrera por ser formadores de las nuevas generaciones de niños y niñas de la educación primaria. Ellos advierten que no solo favorece su formación de pregrado como categoría pedagógica.

Por su parte los docentes durante el análisis desarrollado a partir del trabajo metodológico de la Disciplina Estudios lingüísticos y literarios, corroboraron la significación práctica de la investigación y las posibilidades de promover estudios superiores a través de la superación, los cuales exigían niveles superiores en la calidad de la caligrafía durante la escritura, pues en ocasiones, no tenían en cuenta la importancia para el maestro primario por ser él quien enseña al escolar a escribir y leer. Para ello, la autora principal de la investigación impartió este mismo curso a 30 docentes de las escuelas primarias, donde los estudiantes realizan sus prácticas laborales.

Además, se realizó una entrevista a directivos y docentes que aplicaron la estrategia para la caligrafía corroborándose los siguientes resultados: la estrategia didáctica ha sido útil, necesaria y dinámica, pues posibilitó en tres cursos lograr un salto cualitativo y cuantitativo en la calidad de los estudiantes con respecto a su escritura y caligrafía. También comprendieron la importancia de resolver estas dificultades como futuros maestros de la sociedad. Por su parte, los docentes profundizaron en una temática vital para la formación de maestros primarios en las cuales ellos, en su formación no habían recibido estos contenidos.

Los usuarios corroboraron incluso los propios estudiantes de la carrera, que la enseñanza de la caligrafía en la formación inicial del maestro primario es una necesidad pedagógica profesional, pues ellos son los modelos a imitar. Por tanto, requieren al llegar a la carrera de un reaprendizaje o compensación de sus habilidades y hábitos caligráficos, lo que se logra con un tratamiento diferenciado y consciente tanto de las limitaciones, como de las cualidades caligráficas desde una posición teórico- práctica..

Los docentes son del criterio y en la discusión se evidenció que la evaluación y sistematización de los procesos de aprendizaje y reaprendizaje: las evaluaciones periódicas y sistemáticas que se realizaron aplicando las variantes de las técnicas de trabajo en grupo, promovieron el carácter participativo, democrático, enriquecedor logrando en el proceso pedagógico una excelente comunicación estudiantes- estudiantes 
y estos con sus profesores. Los intercambios de libretas y su revisión correcta permitieron desarrollar habilidades esenciales para el maestro primario.

La implementación del procedimiento elaborado por la autora principal del artículo, Rehabilitación caligráfica prospectiva como una adecuación y aporte al método Análisis Discursivo Funcional del Enfoque cognitivo, comunicativo y sociocultural de Roméu (2006), permitió aplicarse a través de un pre- experimento formativo en la formación inicial de maestros primarios de la Universidad de Guantánamo en la carrera Educación. Primaria.

Desde esta óptica, contextualizar el método Análisis Discursivo Funcional supone asumir la caligrafía como una interacción verbal bella en el discurso escrito y reconocer las tres dimensiones principales (Van Dijk, 2000): el uso del lenguaje (estructura discursiva): el uso de las grafías y su composición- descomposición en rasgos y enlaces, cada uno de ellos en su valor pertinente y distintivo en la sintaxis lo que incide en la semántica.

La grafía es la imagen del fonema, tiene diversos usos según tamaño, forma, color, estilo, perspectiva, grosor y otras categorías relacionadas con las artes visuales, que pueden ser aprendidas por el maestro en formación, para mejorar su desempeño lingüísticometodológico en el ejercicio de la profesión. Al estudiar los estilos como modos socialmente significativos y socialmente interpretados, la variación estilística se comprende como un recurso de suma importancia en la interacción escrita.

La comunicación de ideas, creencias (cognición): los conocimientos sobre la caligrafía desde lo anatomofisiológico, histórico- cultural, sociolingüístico, pictórico, y otros, permite mejorar la producción y comunicación de las ideas así como su comprensión en el contexto escolar.

La interacción en situaciones de índole social (sociedad): precisa al maestro como comunicador por excelencia en los diferentes contextos de actuación que le plantea la praxis pedagógica. La grafía es la letra que enlazada constituye la cadena textual escrita y si el término texto implica tanto el discurso oral como el escrito, vistos ambos en su relación con el contexto, esta y sus cualidades deben ser consideradas en el análisis discursivo y en la producción resultante.

El procedimiento Rehabilitación caligráfica prospectiva, se define como "el conjunto de acciones instructivas, educativas, de formación profesional de tiempo limitado, articuladas, definidas y conducidas por un equipo interdisciplinario". (Lescaille; 2018:) 
El procedimiento moviliza al usuario como sujeto activo de su propio proceso, a la familia, a la comunidad laboral y a la comunidad social, agentes educativos de la escuela lo que promueve el cumplimiento de los objetivos trazados, y los cambios en el docente, sus estudiantes y en el entorno educativo lo cual conduce a un reaprendizaje de la caligrafía, al desarrollo de un proceso cognitivo satisfactorio y a una actuación profesional con calidad.

Los docentes que participaron como muestra y población de la investigación corroboran que el procedimiento se connota como el despliegue de acciones y operaciones a realizar por el profesor y el maestro primario en formación inicial, que conducen a un estudio específico condicionado de las grafías, los rasgos que las componen y las cualidades que deben tratarse para el logro de la melodía cinética. Además, conduce a la armonía de la realización práctica como una secuencia concertada de los impulsos psico y neuromotrices expresados en el estilo cursivo; cuyo empleo conduce a una solución que involucra la asimilación, el acomodamiento y la compensación, lo cual controla el logro de una actuación caligráfica competente. O sea, consiste en la ejecución consciente sistemática de acciones con el objetivo de adquirir conocimientos más significativos llevados a la práctica a través de situaciones nuevas.

Todos los participantes en la experiencia participativa enfatizan que su estructura interna, lo que operacionaliza la actuación desde la unidad pensamiento comunicativo, y que aunque los procedimientos y los métodos son parte de la didáctica, son generadores para el desarrollo de la formación de egresados. Por tanto, no es posible perder de vista la relación entre estas ciencias de la educación. A partir de la instrucción y la educación como categorías pedagógicas se exponen las operaciones para el trabajo con las grafías.

- Acondicionamiento higiénico, ordenamiento del espacio y los medios

- Observación reflexiva de las grafías.

- Verbalización de sus particularidades: forma, rasgos y enlaces.

- Reflexión sobre los modos de actuación psico y verbo motriz mediatizado por las relaciones y coordinaciones viso espaciales.

- Reflexión de uso según fin comunicativo o intención (permite considerar la creatividad: estilo, tamaño, color, perspectiva, etc.)

- Realización del proceso de escritura.

- Autorrevisión 


\section{Operaciones para el logro de las cualidades}

Los docentes después de su preparación acerca del tema de la caligrafía, comprenden que las cualidades caligráficas tienen sus operaciones específicas. De modo general su logro pasa por la observación, la reflexión estética que incluye los requisitos de cada una y que en su conjunto contribuyen a la armonía como cualidad máxima de la caligrafía. La propuesta de operacionalización de las cualidades que se trabajan en la escuela primaria son: corrección, uniformidad, inclinación y fluidez. También pueden tenerse en cuenta, el ritmo, grosor, color, tamaño, presión y sobre todo el espacio entre caracteres y entre segmentos léxicos.

Ello implica el desarrollo de estrategias para facilitar su asimilación donde el profesor responde a las interrogantes: ¿Qué conozco de la caligrafía?; ¿Qué indicaciones debo seguir para dirigir el proceso de enseñanza- aprendizaje? ¿Qué operaciones verbo motrices, debo activar para el trazado y enlace de las grafías en particular y de una secuencia gráfica en general? y ¿En qué condiciones metodológicas me encuentro?

Por su parte, el maestro primario en formación inicial, responde a las interrogantes: ¿qué tipo de escritura necesito asimilar?; ¿cómo debo proceder para su asimilación?, ¿cómo procedo críticamente ante situaciones cognoscitivas específicas que debo enfrentar para el reaprendizaje?; ¿qué actitud debo asumir para llegar al resultado esperado? El procedimiento expresado se usa en situaciones en las cuales, se requiere una secuencia de acciones que conduzcan a una transformación que corrija o compense las deformaciones en el actuar caligráfico.

Este procedimiento, se apoya en el método fónico- analítico- sintético, y el viso- audiognósico- motor de gran tradición en la enseñanza de la lengua materna en la escuela primaria, que permite fortalecer el método Análisis Discursivo Funcional para la Educación Superior, lo que constituye punto de partida para su concepción.

Todos coinciden en el análisis del impacto e instrumentación del procedimiento Rehabilitación caligráfica prospectiva, que posee una estructura interna, se organiza por acciones ordenadas, desde la integración de habilidades verbo-motrices y psicomotrices en el proceso de enseñanza - aprendizaje. Dichas acciones dependen de la orientación cognitiva contextualizada para el alcance de una sistematización metodológica, lo que favorece la autorrevisión eficiente del proceso, y un despliegue comunicativo expresivo en el plano escrito. Al mismo tiempo, conducen a una actuación profesional y al 
desarrollo del estudiante. Las valoraciones realizadas con la implementación de la estrategia y la contextualización del método y su procedimiento permiten elaborar las siguientes conclusiones.

\section{CONCLUSIONES}

La estrategia pedagógica para la caligrafía es la concreción práctica de la investigación, su implementación y valoración en la praxis pedagógica de formación de profesionales con calidad para la educación primaria, ha posibilitado constatar el salto cualitativo y cuantitativo logrado por los estudiantes y el grado de satisfacción de directivos y docentes de la carrera.

Los profesores y especialistas que han sido muestra y usuarios en la aplicación de la propuesta, ofrecen una valoración positiva al destacar lo pertinente, necesario y útil del aporte práctico destacando su correspondencia con el perfil ocupacional del maestro y su contribución al desarrollo del pensamiento lógico a partir del desarrollo del pensamiento comunicativo y del pensamiento estético.

El procedimiento propuesto constituye una herramienta pedagógica y didáctico metodológica de apoyo a los profesores en la conducción del proceso pedagógico y del proceso de enseñanza- aprendizaje de la caligrafía, porque posibilita la operacionalización como base orientadora de la actividad para al maestro primario en formación, permitiéndoles la asimilación de nuevos saberes en el reaprendizaje de la caligrafía, al compensar sus dificultades y acomodarlos a la nueva actuación en su desempeño profesional.

\section{REFERENCIAS BIBLIOGRÁFICAS}

Domínguez, I. (2003). La normativa caligráfica. La Habana. Editorial Pueblo y Educación.

Fariñas. G (1996). Maestros, una estrategia para la enseñanza. Revista Maestro y sociedad. Vol ( 7) pp.(19-24)

García Gilberto et al. (2004.). Profesionalismo y práctica pedagógica. La Habana. Editorial Pueblo y Educación. pp. 77- 93.

González B, Susana. (2008). La escritura en la escuela. Recuperado en: http://www.educar.org/articulos/escritura.asp 
Lescaille Rivera, M. (2012). Actividades docentes para el tratamiento a la caligrafía en la formación inicial del maestro primario. (Tesis impresa de maestría) Universidad de Ciencias Pedagógicas. Guantánamo. Cuba.

Lescaille. Rivera, M (2015). La caligrafía como habilidad profesional pedagógica. Revista Edusol. Vol 2. \# 4. pp. 23- 27.

Lescaille. Rivera, M (2019). La caligrafía en la formación del maestro primario. (tesis doctoral inédita). Universidad de Oriente. Santiago de Cuba- Cuba.

Luria, A. (1982). El cerebro en acción. (2da. Edición). La Habana. Editorial Pueblo y Educación.

Roméu, A. (2008). El enfoque cognitivo-comunicativo y sociocultural en la enseñanza de la lengua y la literatura. La Habana. Editorial Pueblo y Educación.

Torres. M. (2004). El diagnóstico psicopedagógico. Conferencia en el III Congreso de Educación Especial. La Habana.

Vygotsky S. L. (1982). Pensamiento y lenguaje. La Habana. Editorial Pueblo y Educación. 